\title{
Convergent-Beam Electron Diffraction Study of Local Structural Fluctuations in Perovskite-Type Ferroelectrics
}

\author{
Kenji Tsuda
}

Institute of Multidisciplinary Research for Advanced Materials, Tohoku University, Sendai, Japan

Perovskite-type ferroelectrics have been attracting special attention because of their intriguing physical properties as well as industrial importance. $\mathrm{BaTiO}_{3}$, a typical example of perovskite-type ferroelectrics, undergoes successive phase transformations from the cubic paraelectric phase to three ferroelectric phases: tetragonal, orthorhombic and rhombohedral ones. Coexistence of the displacive and orderdisorder characters in the phase transformations of $\mathrm{BaTiO}_{3}$ was pointed out from many experiments and theories [ex. 1, 2]. However, local structures related to the order-disorder character were observed neither in crystal structure analyses using neutron and X-ray diffraction nor by TEM observations. In this study, we applied the convergent-beam electron diffraction (CBED) method, which is a most powerful technique for local symmetry determination $[3,4]$, to examine nanometer-scale local structures of perovskite-type ferroelectrics.

Using CBED, we observed that the orthorhombic and tetragonal phases of $\mathrm{BaTiO}_{3}$ have rhombohedral nanostructures [5]. We also found that the symmetry of the orthorhombic phase is formed as the average of two rhombohedral variants with different polarizations, and that of the tetragonal phase is formed as the average of four rhombohedral variants. These indicate an order-disorder character in their phase transformations. Similar rhombohedral local structures were observed in the ferroelectric orthorhombic phase of $\mathrm{KNbO}_{3}$ [6], while the local structure of the ferroelectric tetragonal phase of $\mathrm{PbTiO}_{3}$ was revealed to have the same tetragonal symmetry as the average structure [7].

To examine distributions of the nanostructures and local polarizations, we proposed a combined use of STEM and CBED methods (STEM-CBED method) [8]. Figure 1 shows a schematic diagram of the STEM-CBED method. CBED patterns are acquired pixel-by-pixel by scanning the convergent-beam electron probe with a sub-nanometer step. Accurate control of the incident beam direction and selecting specimen areas without local bending are necessary for correctly detecting symmetry breaking of CBED patterns. A similar method using cross-correlation coefficients was proposed by Kim et al.[9]. Using the STEM-CBED method, two-dimensional distributions of the rhombohedral nanostructures, or nanoscale fluctuations of polarization clusters, were successfully visualized in the tetragonal phase of $\mathrm{BaTiO}_{3}$ [8].

Figure 2 shows a STEM-CBED map and an energy-filtered CBED pattern of the orthorhombic phase of $\mathrm{KNbO}_{3}$ taken at the $[001]_{\mathrm{PC}}$ incidence, where the scan step was set to $0.5 \mathrm{~nm}$. From the acquired CBED patterns, intensity differences between the $1-10$ PC and - $-1-10$ PC reflections were mapped. Since these reflections are symmetrically equivalent in the orthorhombic phase, the map indicates breakdown of the orthorhombic symmetry and their nanometer-scale fluctuations. (The above indices of the direction and reflections are expressed by the pseudocubic (PC) axes.)

The study was performed in collaboration with Emer. Prof. M. Tanaka and Mr. R. Sano (IMRAM, Tohoku University), and Mr. A. Yasuhara (JEOL Ltd.), and was supported by JSPS KAKENHI Grant Numbers 25287068 and 256630272. Prof. M. Terauchi is thanked for his support to this work. 
References:

[1] R. Pirc and R. Blinc, Phys. Rev. B 70 (2004) 134107.

[2] G. Völkel and K. A. Müller, Phys. Rev. B 76 (2007) 094105.

[3] M. Tanaka and K. Tsuda, J. Electron Microsc. 60(Suppl. 1) (2011) S245.

[4] Y. Shi et al., Nature Mat., 12 (2013) p. 1024.

[5] K. Tsuda, R. Sano and M. Tanaka, Phys. Rev. B 86 (2012) 214106.

[6] K. Tsuda, R. Sano and M. Tanaka, Appl. Phys. Lett. 102 (2013) 051913.

[7] K. Tsuda and M. Tanaka, Appl. Phys. Express 6 (2013) 101501.

[8] K. Tsuda, A. Yasuhara and M. Tanaka, Appl. Phys. Lett. 102 (2013) 051913.

[9] K. H. Kim, D. A. Payne and J. M. Zuo, J. Appl. Cryst. 46 (2013) p. 1331.

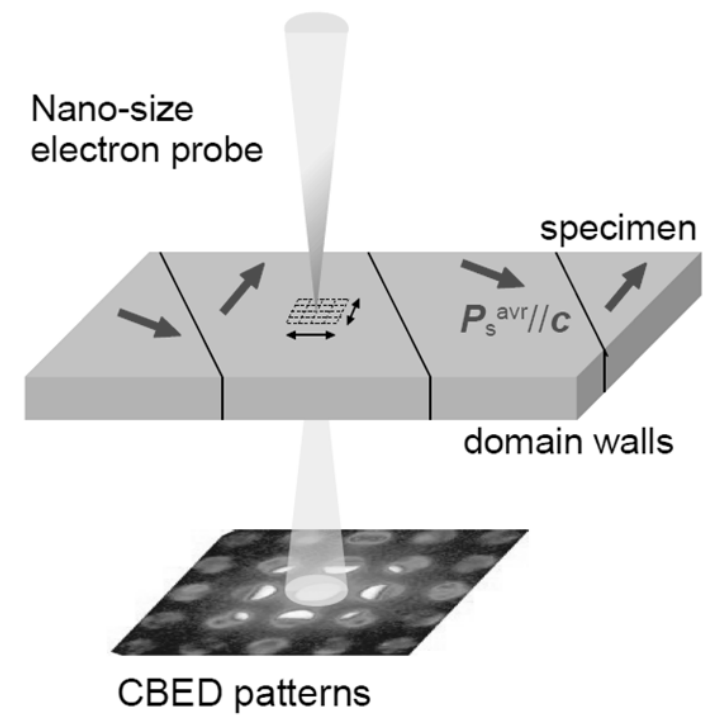

Figure 1. Schematic diagram of the STEM-CBED method.

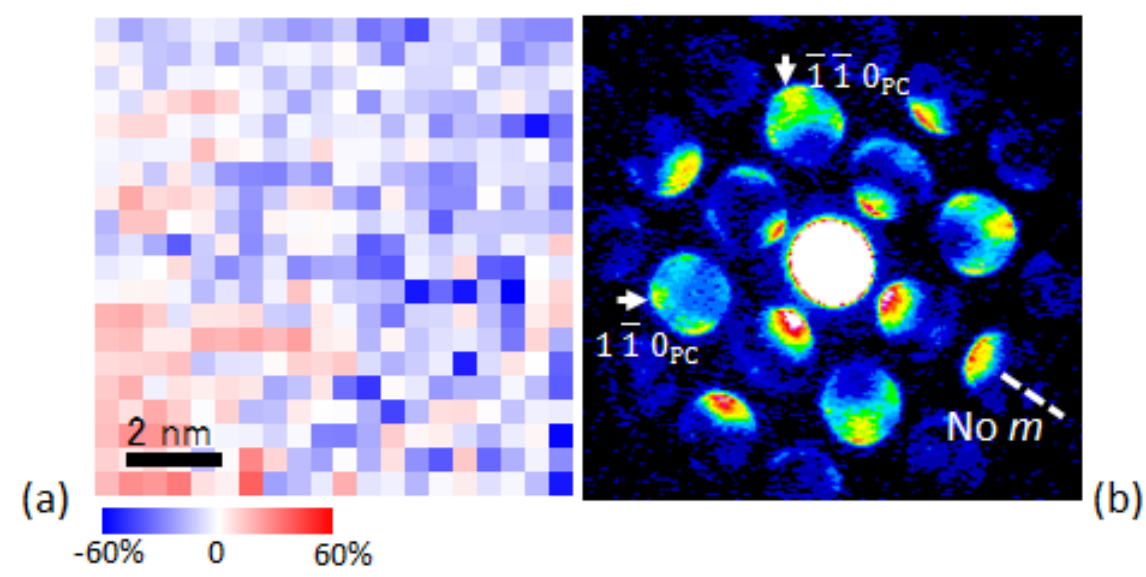

Figure 2. (a) STEM-CBED map of the orthorhombic phase of $\mathrm{KNbO}_{3}$ and (b) energy-filtered CBED pattern taken at the $[001]_{\mathrm{PC}}$ incidence (PC: pseudocubic). The STEM-CBED map shows the intensity difference between the $1-10$ PC and $-1-10$ PC reflections, ( $I_{1-1} 0$ PC $-I_{-1-1} 0$ PC $) / I_{-1-1} 0$ PC, which is colored with the attached color bar for a range of $-60 \%$ to $60 \%$. 